\title{
Stability of general multi-Euler-Lagrange quadratic functional equations in non-Archimedean fuzzy normed spaces
}

Tian Zhou $\mathrm{Xu}^{1 *}$ and John Michael Rassias ${ }^{2}$

"Correspondence: xutianzhou@bit.edu.cn ${ }^{1}$ School of Mathematics, Beijing Institute of Technology, Beijing, 100081, P.R. China

Full list of author information is available at the end of the article

\begin{abstract}
In this paper we prove the generalized Hyers-Ulam stability of the system defining general Euler-Lagrange quadratic mappings in non-Archimedean fuzzy normed spaces over a field with valuation using the direct and the fixed point methods. MSC: 39B82; 39B52; 46H25
\end{abstract}

Keywords: stability of general multi-Euler-Lagrange quadratic functional equation; direct method; fixed point method; non-Archimedean fuzzy normed space

\section{Introduction}

Let $\mathbb{K}$ be a field. A valuation mapping on $\mathbb{K}$ is a function $|\cdot|: \mathbb{K} \rightarrow \mathbb{R}$ such that for any $r, s \in \mathbb{K}$ the following conditions are satisfied: (i) $|r| \geq 0$ and equality holds if and only if $r=0$; (ii) $|r s|=|r| \cdot|s|$; (iii) $|r+s| \leq|r|+|s|$.

A field endowed with a valuation mapping will be called a valued field. The usual absolute values of $\mathbb{R}$ and $\mathbb{C}$ are examples of valuations. A trivial example of a non-Archimedean valuation is the function $|\cdot|$ taking everything except for 0 into 1 and $|0|=0$. In the following we will assume that $|\cdot|$ is non-trivial, i.e., there is an $r_{0} \in \mathbb{K}$ such that $\left|r_{0}\right| \neq 0,1$.

If the condition (iii) in the definition of a valuation mapping is replaced with a strong triangle inequality (ultrametric): $|r+s| \leq \max \{|r|,|s|\}$, then the valuation $|\cdot|$ is said to be non-Archimedean. In any non-Archimedean field we have $|1|=|-1|=1$ and $|n| \leq 1$ for all $n \in \mathbb{N}$.

Throughout this paper, we assume that $\mathbb{K}$ is a valued field, $\mathcal{X}$ and $\mathcal{Y}$ are vector spaces over $\mathbb{K}, a, b \in \mathbb{K}$ are fixed with $\lambda:=a^{2}+b^{2} \neq 0,1\left(\lambda_{1}:=2 a \neq 0,1\right.$ if $\left.a=b\right)$ and $n$ is a positive integer. Moreover, $\mathbb{N}$ stands for the set of all positive integers and $\mathbb{R}$ (respectively, $\mathbb{Q}$ ) denotes the set of all reals (respectively, rationals).

A mapping $f: \mathcal{X}^{n} \rightarrow \mathcal{Y}$ is called a general multi-Euler-Lagrange quadratic mapping if it satisfies the general Euler-Lagrange quadratic equations in each of their $n$ arguments:

$$
\begin{aligned}
& f\left(x_{1}, \ldots, x_{i-1}, a x_{i}+b x_{i}^{\prime}, x_{i+1}, \ldots, x_{n}\right)+f\left(x_{1}, \ldots, x_{i-1}, b x_{i}-a x_{i}^{\prime}, x_{i+1}, \ldots, x_{n}\right) \\
& \quad=\left(a^{2}+b^{2}\right)\left[f\left(x_{1}, \ldots, x_{n}\right)+f\left(x_{1}, \ldots, x_{i-1}, x_{i}^{\prime}, x_{i+1}, \ldots, x_{n}\right)\right]
\end{aligned}
$$

(c) 2012 Xu and Rassias; licensee Springer. This is an Open Access article distributed under the terms of the Creative Commons Attribution License (http://creativecommons.org/licenses/by/2.0), which permits unrestricted use, distribution, and reproduction in any medium, provided the original work is properly cited. 
for all $i=1, \ldots, n$ and all $x_{1}, \ldots, x_{i-1}, x_{i}, x_{i}^{\prime}, x_{i+1}, \ldots, x_{n} \in \mathcal{X}$. Letting $x_{i}=x_{i}^{\prime}=0$ in (1.1), we get $f\left(x_{1}, \ldots, x_{i-1}, 0, x_{i+1}, \ldots, x_{n}\right)=0$. Putting $x_{i}^{\prime}=0$ in (1.1), we have

$$
f\left(x_{1}, \ldots, x_{i-1}, a x_{i}, x_{i+1}, \ldots, x_{n}\right)+f\left(x_{1}, \ldots, x_{i-1}, b x_{i}, x_{i+1}, \ldots, x_{n}\right)=\lambda f\left(x_{1}, \ldots, x_{n}\right) .
$$

Replacing $x_{i}$ by $a x_{i}$ and $x_{i}^{\prime}$ by $b x_{i}$ in (1.1), respectively, we obtain

$$
\begin{aligned}
& f\left(x_{1}, \ldots, x_{i-1}, \lambda x_{i}, x_{i+1}, \ldots, x_{n}\right) \\
& \quad=\lambda\left[f\left(x_{1}, \ldots, x_{i-1}, a x_{i}, x_{i+1}, \ldots, x_{n}\right)+f\left(x_{1}, \ldots, x_{i-1}, b x_{i}, x_{i+1}, \ldots, x_{n}\right)\right] .
\end{aligned}
$$

From (1.2) and (1.3), one gets

$$
f\left(x_{1}, \ldots, x_{i-1}, \lambda x_{i}, x_{i+1}, \ldots, x_{n}\right)=\lambda^{2} f\left(x_{1}, \ldots, x_{n}\right)
$$

for all $i=1, \ldots, n$ and all $x_{1}, \ldots, x_{n} \in \mathcal{X}$. If $a=b$ in (1.1), then we have

$$
\begin{aligned}
& f\left(x_{1}, \ldots, x_{i-1}, a\left(x_{i}+x_{i}^{\prime}\right), x_{i+1}, \ldots, x_{n}\right)+f\left(x_{1}, \ldots, x_{i-1}, a\left(x_{i}-x_{i}^{\prime}\right), x_{i+1}, \ldots, x_{n}\right) \\
& \quad=2 a^{2}\left[f\left(x_{1}, \ldots, x_{n}\right)+f\left(x_{1}, \ldots, x_{i-1}, x_{i}^{\prime}, x_{i+1}, \ldots, x_{n}\right)\right] .
\end{aligned}
$$

Letting $x_{i}^{\prime}=x_{i}$ in (1.5), we obtain

$$
f\left(x_{1}, \ldots, x_{i-1}, \lambda_{1} x_{i}, x_{i+1}, \ldots, x_{n}\right)=\lambda_{1}^{2} f\left(x_{1}, \ldots, x_{n}\right)
$$

for all $i=1, \ldots, n$ and all $x_{1}, \ldots, x_{n} \in \mathcal{X}$.

The study of stability problems for functional equations is related to a question of Ulam [30] concerning the stability of group homomorphisms and affirmatively answered for Banach spaces by Hyers [13]. The result of Hyers was generalized by Aoki [2] for approximate additive mappings and by Rassias [27] for approximate linear mappings by allowing the Cauchy difference operator $C D f(x, y)=f(x+y)-[f(x)+f(y)]$ to be controlled by $\epsilon\left(\|x\|^{p}+\|y\|^{p}\right)$. In 1994, a further generalization was obtained by Găvruța [9], who replaced $\epsilon\left(\|x\|^{p}+\|y\|^{p}\right)$ by a general control function $\varphi(x, y)$. We refer the reader to see, for instance, $[1,4-7,14-16,18,20,22,23,25,26,28,31-37]$ for more information on different aspects of stability of functional equations. On the other hand, for some outcomes on the stability of multi-quadratic and Euler-Lagrange-type quadratic mappings we refer the reader to [7, 11, 24].

The main purpose of this paper is to prove the generalized Hyers-Ulam stability of multiEuler-Lagrange quadratic functional equation (1.1) in complete non-Archimedean fuzzy normed spaces over a field with valuation using the direct and the fixed point methods.

\section{Preliminaries}

We recall the notion of non-Archimedean fuzzy normed spaces over a field with valuation and some preliminary results (see for instance [3, 22, 23, 31, 32]). For more details the reader is referred to $[3,22]$.

Definition 2.1 Let $\mathcal{X}$ be a linear space over a field $\mathbb{K}$ with a non-Archimedean valuation $|\cdot|$. A function $\|\cdot\|: \mathcal{X} \rightarrow[0, \infty)$ is said to be a non-Archimedean norm if it satisfies the following conditions: 
(i) $\|x\|=0$ if and only if $x=0$;

(ii) $\|r x\|=|r|\|x\|, r \in \mathbb{K}, x \in \mathcal{X}$;

(iii) the strong triangle inequality

$$
\|x+y\| \leq \max \{\|x\|,\|y\|\}, \quad x, y \in \mathcal{X}
$$

Then $(\mathcal{X},\|\cdot\|)$ is called a non-Archimedean normed space. By a complete non-Archimedean normed space, we mean one in which every Cauchy sequence is convergent.

In 1897, Hensel discovered the $p$-adic numbers as a number-theoretical analogue of power series in complex analysis. Let $p$ be a prime number. For any nonzero rational number $a$, there exists a unique integer $r$ such that $a=p^{r} m / n$, where $m$ and $n$ are integers not divisible by $p$. Then $|a|_{p}:=p^{-r}$ defines a non-Archimedean norm on $\mathbb{Q}$. The completion of $\mathbb{Q}$ with respect to the metric $d(a, b)=|a-b|_{p}$ is denoted by $\mathbb{Q}_{p}$ which is called the $p$-adic number field. Note that if $p>2$, then $\left|2^{n}\right|_{p}=1$ for each integer $n$ but $|2|_{2}<1$.

During the last three decades, $p$-adic numbers have gained the interest of physicists for their research, in particular, into problems deriving from quantum physics, $p$-adic strings, and superstrings (see for instance [21]).

A triangular norm (shorter $t$-norm, [29]) is a binary operation $T:[0,1] \times[0,1] \rightarrow$ $[0,1]$ which satisfies the following conditions: (a) $T$ is commutative and associative; (b) $T(a, 1)=a$ for all $a \in[0,1]$; (c) $T(a, b) \leq T(c, d)$ whenever $a \leq c$ and $b \leq d$ for all $a, b, c, d \in[0,1]$. Basic examples of continuous $t$-norms are the Łukasiewicz $t$-norm $T_{L}$, $T_{L}(a, b)=\max \{a+b-1,0\}$, the product $t$-norm $T_{P}, T_{P}(a, b)=a b$ and the strongest triangular norm $T_{M}, T_{M}(a, b)=\min \{a, b\}$. A $t$-norm is called continuous if it is continuous with respect to the product topology on the set $[0,1] \times[0,1]$.

A $t$-norm $T$ can be extended (by associativity) in a unique way to an $m$-array operation taking for $\left(x_{1}, \ldots, x_{m}\right) \in[0,1]^{m}$, the value $T\left(x_{1}, \ldots, x_{m}\right)$ defined recurrently by $T_{i=1}^{0} x_{i}=1$ and $T_{i=1}^{m} x_{i}=T\left(T_{i=1}^{m-1} x_{i}, x_{m}\right)$ for $m \in \mathbb{N}$. $T$ can also be extended to a countable operation, taking for any sequence $\left\{x_{i}\right\}_{i \in \mathbb{N}}$ in $[0,1]$, the value $T_{i=1}^{\infty} x_{i}$ is defined as $\lim _{m \rightarrow \infty} T_{i=1}^{m} x_{i}$. The limit exists since the sequence $\left\{T_{i=1}^{m} x_{i}\right\}_{m \in \mathbb{N}}$ is non-increasing and bounded from below. $T_{i=m}^{\infty} x_{i}$ is defined as $T_{i=1}^{\infty} x_{m+i}$.

Definition 2.2 A $t$-norm $T$ is said to be of Hadžić-type ( $H$-type, we denote by $T \in \mathcal{H}$ ) if a family of functions $\left\{T_{i=1}^{m}(t)\right\}$ for all $m \in \mathbb{N}$ is equicontinuous at $t=1$, that is, for all $\varepsilon \in(0,1)$ there exists $\delta \in(0,1)$ such that

$$
t>1-\delta \quad \Longrightarrow \quad T_{i=1}^{m}(t)>1-\varepsilon \quad \text { for all } m \in \mathbb{N} \text {. }
$$

The $t$-norm $T_{M}$ is a $t$-norm of Hadžić-type. Other important triangular norms we refer the reader to [12].

Proposition 2.3 (see [12]) (1) If $T=T_{P}$ or $T=T_{L}$, then

$$
\lim _{m \rightarrow \infty} T_{i=1}^{\infty} x_{m+i}=1 \Longleftrightarrow \sum_{i=1}^{\infty}\left(1-x_{i}\right)<\infty
$$


(2) If $T$ is of Hadžić-type, then

$$
\lim _{m \rightarrow \infty} T_{i=m}^{\infty} x_{i}=\lim _{m \rightarrow \infty} T_{i=1}^{\infty} x_{m+i}=1
$$

for every sequence $\left\{x_{i}\right\}_{i \in \mathbb{N}}$ in $[0,1]$ such that $\lim _{i \rightarrow \infty} x_{i}=1$.

Definition 2.4 (see [22]) Let $\mathcal{X}$ be a linear space over a valued field $\mathbb{K}$ and $T$ be a continuous $t$-norm. A function $N: \mathcal{X} \times \mathbb{R} \rightarrow[0,1]$ is said to be a non-Archimedean fuzzy Menger norm on $\mathcal{X}$ if for all $x, y \in \mathcal{X}$ and all $s, t \in \mathbb{R}$ :

(N1) $N(x, t)=0$ for all $t \leq 0$;

(N2) $x=0$ if and only if $N(x, t)=1, t>0$;

(N3) $N(c x, t)=N(x, t /|c|)$ if $c \neq 0$;

(N4) $N(x+y, \max \{s, t\}) \geq T(N(x, s), N(y, t)), s, t>0$;

(N5) $\lim _{t \rightarrow \infty} N(x, t)=1$.

If $N$ is a non-Archimedean fuzzy Menger norm on $\mathcal{X}$, then the triple $(\mathcal{X}, N, T)$ is called a non-Archimedean fuzzy normed space. It should be noticed that from the condition (N4) it follows that

$$
N(x, t) \geq T(N(0, t), N(x, s))=N(x, s)
$$

for every $t>s>0$ and $x, y \in \mathcal{X}$, that is, $N(x, \cdot)$ is non-decreasing for every $x$. This implies $N(x, s+t) \geq N(x, \max \{s, t\})$. If (N4) holds, then so does

(N6) $N(x+y, s+t) \geq T(N(x, s), N(y, t))$.

We repeatedly use the fact $N(-x, t)=N(x, t), x \in \mathcal{X}, t>0$, which is deduced from (N3). We also note that Definition 2.4 is more general than the definition of a non-Archimedean Menger norm in $[23,31]$, where only fields with a non-Archimedean valuation have been considered.

Definition 2.5 Let $(\mathcal{X}, N, T)$ be a non-Archimedean fuzzy normed space. Let $\left\{x_{m}\right\}_{m \in \mathbb{N}}$ be a sequence in $\mathcal{X}$. Then $\left\{x_{m}\right\}_{m \in \mathbb{N}}$ is said to be convergent if there exists $x \in \mathcal{X}$ such that $\lim _{m \rightarrow \infty} N\left(x_{m}-x, t\right)=1$ for all $t>0$. In that case, $x$ is called the limit of the sequence $\left\{x_{m}\right\}_{m \in \mathbb{N}}$ and we denote it by $\lim _{m \rightarrow \infty} x_{m}=x$. The sequence $\left\{x_{m}\right\}_{m \in \mathbb{N}}$ in $\mathcal{X}$ is said to be a Cauchy sequence if $\lim _{m \rightarrow \infty} N\left(x_{m+p}-x_{m}, t\right)=1$ for all $t>0$ and $p=1,2, \ldots$ If every Cauchy sequence in $\mathcal{X}$ is convergent, then the space is called a complete non-Archimedean fuzzy normed space.

Example 2.6 Let $(\mathcal{X},\|\cdot\|)$ be a real (or non-Archimedean) normed space. For each $k>0$, consider

$$
N_{k}(x, t)= \begin{cases}\frac{t}{t+k\|x\|}, & t>0 \\ 0, & t \leq 0\end{cases}
$$

Then $\left(\mathcal{X}, N_{k}, T_{M}\right)$ is a non-Archimedean fuzzy normed space.

Example 2.7 (see [22]) Let $(\mathcal{X},\|\cdot\|)$ be a real normed space. Then the triple $\left(\mathcal{X}, N, T_{P}\right)$, 
where

$$
N(x, t)= \begin{cases}e^{-\|x\| / t}, & t>0, \\ 0, & t \leq 0\end{cases}
$$

is a non-Archimedean fuzzy normed space. Moreover, if $(\mathcal{X},\|\cdot\|)$ is complete, then $\left(\mathcal{X}, N, T_{P}\right)$ is complete and therefore it is a complete non-Archimedean fuzzy normed space over an Archimedean valued field.

Let $\Omega$ be a set. A function $d: \Omega \times \Omega \rightarrow[0, \infty]$ is called a generalized metric on $\Omega$ if $d$ satisfies

(1) $d(x, y)=0$ if and only if $x=y$; (2) $d(x, y)=d(y, x), x, y \in \Omega$; (3) $d(x, y) \leq d(x, z)+d(y, z)$, $x, y, z \in \Omega$.

For explicitly later use, we recall the following result by Diaz and Margolis [8].

Theorem 2.8 Let $(\Omega, d)$ be a complete generalized metric space and $J: \Omega \rightarrow \Omega$ be a strictly contractive mapping with Lipschitz constant $0<L<1$, that is

$$
d(J x, J y) \leq L d(x, y), \quad x, y \in \Omega .
$$

If there exists a nonnegative integer $m_{0}$ such that $d\left(J^{m_{0}} x, J^{m_{0}+1} x\right)<\infty$ for an $x \in \Omega$, then

(1) the sequence $\left\{J^{m} x\right\}_{m \in \mathbb{N}}$ converges to a fixed point $x^{*}$ of $J$;

(2) $x^{*}$ is the unique fixed point of $J$ in the set $\Omega$,"

$$
\Omega^{*}:=\left\{y \in \Omega \mid d\left(J^{m_{0}} x, y\right)<\infty\right\} ;
$$

(3) if $y \in \Omega^{*}$, then

$$
d\left(y, x^{*}\right) \leq \frac{1}{1-L} d(y, J y) .
$$

\section{Stability of the functional equation (1.1): a direct method}

Throughout this section, using a direct method, we prove the stability of Eq. (1.1) in complete non-Archimedean fuzzy normed spaces.

Theorem 3.1 Let $\mathbb{K}$ be a valued field, $\mathcal{X}$ be a vector space over $\mathbb{K}$ and $(\mathcal{Y}, N, T)$ be a complete non-Archimedean fuzzy normed space over $\mathbb{K}$. Assume also that, for every $i \in\{1,2, \ldots, n\}, \Psi_{i}: \mathcal{X}^{n+1} \times[0, \infty) \rightarrow[0,1]$ is a mapping such that

$$
\begin{aligned}
& \lim _{j \rightarrow \infty} \Psi_{i}\left(\lambda^{j} x_{1}, \ldots, x_{i}, x_{i}^{\prime}, x_{i+1}, \ldots, x_{n},|\lambda|^{2 j} t\right) \\
&= \cdots \\
&= \lim _{j \rightarrow \infty} \Psi_{i}\left(x_{1}, \ldots, x_{i-2}, \lambda^{j} x_{i-1}, x_{i}, x_{i}^{\prime}, x_{i+1}, \ldots, x_{n},|\lambda|^{2 j} t\right) \\
&= \lim _{j \rightarrow \infty} \Psi_{i}\left(x_{1}, \ldots, x_{i-1}, \lambda^{j} x_{i}, \lambda^{j} x_{i}^{\prime}, x_{i+1}, \ldots, x_{n},|\lambda|^{2 j} t\right) \\
&=\lim _{j \rightarrow \infty} \Psi_{i}\left(x_{1}, \ldots, x_{i}, x_{i}^{\prime}, \lambda^{j} x_{i+1}, x_{i+2}, \ldots, x_{n},|\lambda|^{2 j} t\right)=\ldots \\
&=\lim _{j \rightarrow \infty} \Psi_{i}\left(x_{1}, \ldots, x_{i}, x_{i}^{\prime}, x_{i+1}, \ldots, x_{n-1}, \lambda^{j} x_{n},|\lambda|^{2 j} t\right)=1
\end{aligned}
$$


and

$$
\begin{aligned}
\lim _{k \rightarrow \infty} & T_{j=k}^{\infty} T\left(\Psi_{i}\left(x_{1}, \ldots, x_{i-1}, \lambda^{j} x_{i}, 0, x_{i+1}, \ldots, x_{n},|\lambda|^{2 j+1} t\right),\right. \\
& \left.\Psi_{i}\left(x_{1}, \ldots, x_{i-1}, a \lambda^{j} x_{i}, b \lambda^{j} x_{i}, x_{i+1}, \ldots, x_{n},|\lambda|^{2(j+1)} t\right)\right) \\
= & \lim _{k \rightarrow \infty} T_{j=1}^{\infty} T\left(\Psi_{i}\left(x_{1}, \ldots, x_{i-1}, \lambda^{k+j-1} x_{i}, 0, x_{i+1}, \ldots, x_{n},|\lambda|^{2 k+2 j-1} t\right),\right. \\
& \left.\Psi_{i}\left(x_{1}, \ldots, x_{i-1}, a \lambda^{k+j-1} x_{i}, b \lambda^{k+j-1} x_{i}, x_{i+1}, \ldots, x_{n},|\lambda|^{2 k+2 j} t\right)\right)=1
\end{aligned}
$$

for all $x_{1}, \ldots, x_{i}, x_{i}^{\prime}, x_{i+1}, \ldots, x_{n} \in \mathcal{X}$ and $t>0$. Iff $: \mathcal{X}^{n} \rightarrow \mathcal{Y}$ is a mapping satisfying

$$
f\left(x_{1}, \ldots, x_{i-1}, 0, x_{i+1}, \ldots, x_{n}\right)=0 \text {, }
$$

and

$$
\begin{aligned}
& N\left(f\left(x_{1}, \ldots, x_{i-1}, a x_{i}+b x_{i}^{\prime}, x_{i+1}, \ldots, x_{n}\right)+f\left(x_{1}, \ldots, x_{i-1}, b x_{i}-a x_{i}^{\prime}, x_{i+1}, \ldots, x_{n}\right)\right. \\
& \left.\quad-\left(a^{2}+b^{2}\right)\left[f\left(x_{1}, \ldots, x_{n}\right)+f\left(x_{1}, \ldots, x_{i-1}, x_{i}^{\prime}, x_{i+1}, \ldots, x_{n}\right)\right], t\right) \\
& \geq \Psi_{i}\left(x_{1}, \ldots, x_{i}, x_{i}^{\prime}, x_{i+1}, \ldots, x_{n}, t\right)
\end{aligned}
$$

for all $x_{1}, \ldots, x_{i}, x_{i}^{\prime}, x_{i+1}, \ldots, x_{n} \in \mathcal{X}, i \in\{1,2, \ldots, n\}$ and $t \in[0, \infty)$, then for every $i \in$ $\{1,2, \ldots, n\}$ there exists a unique general multi-Euler-Lagrange quadratic mapping $Q_{i}$ : $\mathcal{X}^{n} \rightarrow \mathcal{Y}$ such that

$$
\begin{aligned}
& N\left(f\left(x_{1}, \ldots, x_{n}\right)-Q_{i}\left(x_{1}, \ldots, x_{n}\right), t\right) \\
& \geq T_{j=1}^{\infty} T\left(\Psi_{i}\left(x_{1}, \ldots, x_{i-1}, \lambda^{j-1} x_{i}, 0, x_{i+1}, \ldots, x_{n},|\lambda|^{2 j-1} t\right),\right. \\
& \left.\quad \Psi_{i}\left(x_{1}, \ldots, x_{i-1}, a \lambda^{j-1} x_{i}, b \lambda^{j-1} x_{i}, x_{i+1}, \ldots, x_{n},|\lambda|^{2 j} t\right)\right)
\end{aligned}
$$

for all $x_{1}, \ldots, x_{n} \in \mathcal{X}$ and $t>0$.

Proof Fix $x_{1}, \ldots, x_{n} \in \mathcal{X}, j \in \mathbb{N} \cup\{0\}, i \in\{1,2, \ldots, n\}$ and $t>0$. Putting $x_{i}^{\prime}=0$ in (3.4), we get

$$
\begin{aligned}
& N\left(f\left(x_{1}, \ldots, x_{i-1}, a x_{i}, x_{i+1}, \ldots, x_{n}\right)+f\left(x_{1}, \ldots, x_{i-1}, b x_{i}, x_{i+1}, \ldots, x_{n}\right)\right. \\
& \left.\quad-\lambda f\left(x_{1}, \ldots, x_{n}\right), t\right) \geq \Psi_{i}\left(x_{1}, \ldots, x_{i}, 0, x_{i+1}, \ldots, x_{n}, t\right) .
\end{aligned}
$$

Replacing $x_{i}$ by $a x_{i}$ and $x_{i}^{\prime}$ by $b x_{i}$ in (3.4), respectively, we have

$$
\begin{aligned}
& N\left(f\left(x_{1}, \ldots, x_{i-1}, \lambda x_{i}, x_{i+1}, \ldots, x_{n}\right)-\lambda f\left(x_{1}, \ldots, x_{i-1}, a x_{i}, x_{i+1}, \ldots, x_{n}\right)\right. \\
& \left.\quad-\lambda f\left(x_{1}, \ldots, x_{i-1}, b x_{i}, x_{i+1}, \ldots, x_{n}\right), t\right) \geq \Psi_{i}\left(x_{1}, \ldots, x_{i-1}, a x_{i}, b x_{i}, x_{i+1}, \ldots, x_{n}, t\right) .
\end{aligned}
$$

From (3.6) and (3.7), one gets

$$
\begin{aligned}
N( & \left.\frac{1}{\lambda^{2}} f\left(x_{1}, \ldots, x_{i-1}, \lambda x_{i}, x_{i+1}, \ldots, x_{n}\right)-f\left(x_{1}, \ldots, x_{n}\right), t\right) \\
\geq & T\left(\Psi_{i}\left(x_{1}, \ldots, x_{i}, 0, x_{i+1}, \ldots, x_{n},|\lambda| t\right)\right. \\
& \left.\Psi_{i}\left(x_{1}, \ldots, x_{i-1}, a x_{i}, b x_{i}, x_{i+1}, \ldots, x_{n},|\lambda|^{2} t\right)\right) .
\end{aligned}
$$


Therefore one can get

$$
\begin{aligned}
N( & \left.\frac{1}{\lambda^{2(k+p)}} f\left(x_{1}, \ldots, x_{i-1}, \lambda^{k+p} x_{i}, x_{i+1}, \ldots, x_{n}\right)-\frac{1}{\lambda^{2 k}} f\left(x_{1}, \ldots, x_{i-1}, \lambda^{k} x_{i}, x_{i+1}, \ldots, x_{n}\right), t\right) \\
\geq & T_{j=k}^{k+p-1} T\left(\Psi_{i}\left(x_{1}, \ldots, x_{i-1}, \lambda^{j} x_{i}, 0, x_{i+1}, \ldots, x_{n},|\lambda|^{2 j+1} t\right),\right. \\
& \left.\Psi_{i}\left(x_{1}, \ldots, x_{i-1}, a \lambda^{j} x_{i}, b \lambda^{j} x_{i}, x_{i+1}, \ldots, x_{n},|\lambda|^{2(j+1)} t\right)\right),
\end{aligned}
$$

and thus from (3.2) it follows that $\left\{\frac{1}{\lambda^{2 j}} f\left(x_{1}, \ldots, x_{i-1}, \lambda^{j} x_{i}, x_{i+1}, \ldots, x_{n}\right)\right\}_{j \in \mathbb{N}}$ is a Cauchy sequence in a complete non-Archimedean fuzzy normed space. Hence, we can define a mapping $Q_{i}: \mathcal{X}^{n} \rightarrow \mathcal{Y}$ such that

$$
\lim _{j \rightarrow \infty} N\left(\frac{1}{\lambda^{2 j}} f\left(x_{1}, \ldots, x_{i-1}, \lambda^{j} x_{i}, x_{i+1}, \ldots, x_{n}\right)-Q_{i}\left(x_{1}, \ldots, x_{n}\right), t\right)=1 .
$$

Next, for each $k \in \mathbb{N}$ with $k \geq 1$, we have

$$
\begin{gathered}
N\left(f\left(x_{1}, \ldots, x_{n}\right)-\frac{1}{\lambda^{2 k}} f\left(x_{1}, \ldots, x_{i-1}, \lambda^{k} x_{i}, x_{i+1}, \ldots, x_{n}\right), t\right) \\
\geq T_{j=1}^{k} N\left(\frac{1}{\lambda^{2(j-1)}} f\left(x_{1}, \ldots, x_{i-1}, \lambda^{j-1} x_{i}, x_{i+1}, \ldots, x_{n}\right)\right. \\
\left.\quad-\frac{1}{\lambda^{2} j} f\left(x_{1}, \ldots, x_{i-1}, \lambda^{j} x_{i}, x_{i+1}, \ldots, x_{n}\right), t\right) \\
\geq T_{j=1}^{k} T\left(\Psi_{i}\left(x_{1}, \ldots, x_{i-1}, \lambda^{j-1} x_{i}, 0, x_{i+1}, \ldots, x_{n},|\lambda|^{2 j-1} t\right),\right. \\
\left.\Psi_{i}\left(x_{1}, \ldots, x_{i-1}, a \lambda^{j-1} x_{i}, b \lambda^{j-1} x_{i}, x_{i+1}, \ldots, x_{n},|\lambda|^{2 j} t\right)\right) .
\end{gathered}
$$

Therefore,

$$
\begin{aligned}
& N\left(f\left(x_{1}, \ldots, x_{n}\right)-Q_{i}\left(x_{1}, \ldots, x_{n}\right), t\right) \\
& \geq T\left(N\left(f\left(x_{1}, \ldots, x_{n}\right)-\frac{1}{\lambda^{2 k}} f\left(x_{1}, \ldots, x_{i-1}, \lambda^{k} x_{i}, x_{i+1}, \ldots, x_{n}\right), t\right),\right. \\
&\left.N\left(\frac{1}{\lambda^{2 k}} f\left(x_{1}, \ldots, x_{i-1}, \lambda^{k} x_{i}, x_{i+1}, \ldots, x_{n}\right)-Q_{i}\left(x_{1}, \ldots, x_{n}\right), t\right)\right) \\
& \geq T\left(T _ { j = 1 } ^ { k } T \left(\Psi_{i}\left(x_{1}, \ldots, x_{i-1}, \lambda^{j-1} x_{i}, 0, x_{i+1}, \ldots, x_{n},|\lambda|^{2 j-1} t\right),\right.\right. \\
&\left.\Psi_{i}\left(x_{1}, \ldots, x_{i-1}, a \lambda^{j-1} x_{i}, b \lambda^{j-1} x_{i}, x_{i+1}, \ldots, x_{n},|\lambda|^{2 j} t\right)\right), \\
&\left.N\left(\lambda^{-2 k} f\left(x_{1}, \ldots, x_{i-1}, \lambda^{k} x_{i} x_{i+1}, \ldots, x_{n}\right)-Q_{i}\left(x_{1}, \ldots, x_{n}\right), t\right)\right) .
\end{aligned}
$$

Letting $k \rightarrow \infty$ in this inequality, we obtain (3.5). Now, fix also $x_{i}^{\prime} \in \mathcal{X}$, from (3.1) and (3.4) it follows that

$$
\begin{aligned}
N( & Q_{i}\left(x_{1}, \ldots, x_{i-1}, a x_{i}+b x_{i}^{\prime}, x_{i+1}, \ldots, x_{n}\right)+Q_{i}\left(x_{1}, \ldots, x_{i-1}, b x_{i}-a x_{i}^{\prime}, x_{i+1}, \ldots, x_{n}\right) \\
& \left.-\left(a^{2}+b^{2}\right)\left[Q_{i}\left(x_{1}, \ldots, x_{n}\right)+Q_{i}\left(x_{1}, \ldots, x_{i-1}, x_{i}^{\prime}, x_{i+1}, \ldots, x_{n}\right)\right], t\right) \\
\geq & T\left(N \left(Q_{i}\left(x_{1}, \ldots, x_{i-1}, a x_{i}+b x_{i}^{\prime}, x_{i+1}, \ldots, x_{n}\right)\right.\right. \\
& \left.-\lambda^{-2 j} f\left(x_{1}, \ldots, x_{i-1}, \lambda^{j}\left(a x_{i}+b x_{i}^{\prime}\right), x_{i+1}, \ldots, x_{n}\right), t\right),
\end{aligned}
$$




$$
\begin{aligned}
& N\left(Q_{i}\left(x_{1}, \ldots, x_{i-1}, b x_{i}-a x_{i}^{\prime}, x_{i+1}, \ldots, x_{n}\right)\right. \\
& \left.-\lambda^{-2 j} f\left(x_{1}, \ldots, x_{i-1}, \lambda^{j}\left(b x_{i}-a x_{i}^{\prime}\right), x_{i+1}, \ldots, x_{n}\right), t\right), \\
& N\left(-\lambda Q_{i}\left(x_{1}, \ldots, x_{n}\right)+\lambda^{-2 j+1} f\left(x_{1}, \ldots, x_{i-1}, \lambda^{j} x_{i}, x_{i+1}, \ldots, x_{n}\right), t\right), \\
& N\left(-\lambda Q_{i}\left(x_{1}, \ldots, x_{i-1}, x_{i}^{\prime}, x_{i+1}, \ldots, x_{n}\right)+\lambda^{-2 j+1} f\left(x_{1}, \ldots, x_{i-1}, \lambda^{j} x_{i}^{\prime}, x_{i+1}, \ldots, x_{n}\right), t\right), \\
& \Psi_{i}\left(x_{1}, \ldots, x_{i-1}, \lambda^{j} x_{i}, \lambda^{j} x_{i}^{\prime}, x_{i+1}, \ldots, x_{n},|\lambda|^{2 j} t\right) \rightarrow 1 \quad(j \rightarrow \infty) .
\end{aligned}
$$

Next, fix $k \in\{1, \ldots, n\} \backslash\{i\}, x_{k}^{\prime} \in \mathcal{X}$, and assume, without loss of generality, that $k<i$ (the same arguments apply to the case where $k>i$ ). From (3.1) and (3.4), it follows that

$$
\begin{aligned}
N\left(Q_{i}\right. & \left(x_{1}, \ldots, x_{k-1}, a x_{k}+b x_{k}^{\prime}, x_{k+1}, \ldots, x_{n}\right)+Q_{i}\left(x_{1}, \ldots, x_{k-1}, b x_{k}-a x_{k}^{\prime}, x_{k+1}, \ldots, x_{n}\right) \\
& \left.-\left(a^{2}+b^{2}\right)\left[Q_{i}\left(x_{1}, \ldots, x_{n}\right)+Q_{i}\left(x_{1}, \ldots, x_{k-1}, x_{k}^{\prime}, x_{k+1}, \ldots, x_{n}\right)\right], t\right) \\
\geq & T\left(N \left(Q_{i}\left(x_{1}, \ldots, x_{k-1}, a x_{k}+b x_{k}^{\prime}, x_{k+1}, \ldots, x_{n}\right)\right.\right. \\
& \left.-\lambda^{-2 j} f\left(x_{1}, \ldots, x_{k-1}, a x_{k}+b x_{k}^{\prime}, x_{k+1}, \ldots, x_{i-1}, \lambda^{j} x_{i}, x_{i+1}, \ldots, x_{n}\right), t\right), \\
& N\left(Q_{i}\left(x_{1}, \ldots, x_{k-1}, b x_{k}-a x_{k}^{\prime}, x_{k+1}, \ldots, x_{n}\right)\right. \\
& \left.-\lambda^{-2 j} f\left(x_{1}, \ldots, x_{k-1}, b x_{k}-a x_{k}^{\prime}, x_{k+1}, \ldots, x_{i-1}, \lambda^{j} x_{i}, x_{i+1}, \ldots, x_{n}\right), t\right), \\
& N\left(-\lambda Q_{i}\left(x_{1}, \ldots, x_{n}\right)+\lambda^{-2 j+1} f\left(x_{1}, \ldots, x_{i-1}, \lambda^{j} x_{i}, x_{i+1}, \ldots, x_{n}\right), t\right), \\
& N\left(-\lambda Q_{i}\left(x_{1}, \ldots, x_{k-1}, x_{k}^{\prime}, x_{k+1}, \ldots, x_{n}\right)\right. \\
& \left.+\lambda^{-2 j+1} f\left(x_{1}, \ldots, x_{k-1}, x_{k}^{\prime}, x_{k+1}, \ldots, x_{i-1}, \lambda^{j} x_{i}, x_{i+1}, \ldots, x_{n}\right), t\right), \\
& \left.\Psi_{k}\left(x_{1}, \ldots, x_{k}, x_{k}^{\prime}, x_{k+1}, \ldots, x_{i-1}, \lambda^{j} x_{i}, x_{i+1}, \ldots, x_{n},|\lambda|^{2 j} t\right)\right) \rightarrow 1 \quad(j \rightarrow \infty) .
\end{aligned}
$$

Hence the mapping $Q_{i}$ is a general multi-Euler-Lagrange quadratic mapping. Let us finally assume that $Q_{i}^{\prime}: \mathcal{X}^{n} \rightarrow \mathcal{Y}$ is another multi-Euler-Lagrange quadratic mapping satisfying (3.5). Then, by (1.4), (3.5) and (3.2), it follows that

$$
\begin{aligned}
N( & \left.Q_{i}\left(x_{1}, \ldots, x_{n}\right)-Q_{i}^{\prime}\left(x_{1}, \ldots, x_{n}\right), t\right) \\
= & N\left(Q_{i}\left(x_{1}, \ldots, x_{i-1}, \lambda^{k} x_{i}, x_{i+1}, \ldots, x_{n}\right)-Q_{i}^{\prime}\left(x_{1}, \ldots, x_{i-1}, \lambda^{k} x_{i}, x_{i+1}, \ldots, x_{n}\right),|\lambda|^{2 k} t\right) \\
\geq & T\left(T _ { j = 1 } ^ { \infty } T \left(\Psi_{i}\left(x_{1}, \ldots, x_{i-1}, \lambda^{k+j-1} x_{i}, 0, x_{i+1}, \ldots, x_{n},|\lambda|^{2 k+2 j-1} t\right),\right.\right. \\
& \left.\Psi_{i}\left(x_{1}, \ldots, x_{i-1}, a \lambda^{k+j-1} x_{i}, b \lambda^{k+j-1} x_{i}, x_{i+1}, \ldots, x_{n},|\lambda|^{2 k+2 j} t\right)\right), \\
& T_{j=1}^{\infty} T\left(\Psi_{i}\left(x_{1}, \ldots, x_{i-1}, \lambda^{k+j-1} x_{i}, 0, x_{i+1}, \ldots, x_{n},|\lambda|^{2 k+2 j-1} t\right),\right. \\
& \left.\left.\Psi_{i}\left(x_{1}, \ldots, x_{i-1}, a \lambda^{k+j-1} x_{i}, b \lambda^{k+j-1} x_{i}, x_{i+1}, \ldots, x_{n},|\lambda|^{2 k+2 j} t\right)\right)\right) \\
\rightarrow & 1 \quad(k \rightarrow \infty)
\end{aligned}
$$

and therefore $Q_{i}=Q_{i}^{\prime}$.

For $a=b$, we get the following result.

Theorem 3.2 Let $\mathbb{K}$ be a valued field, $\mathcal{X}$ be a vector space over $\mathbb{K}$ and $(\mathcal{Y}, N, T)$ be a complete non-Archimedean fuzzy normed space over $\mathbb{K}$. Assume also that, for every 
$i \in\{1,2, \ldots, n\}, \Psi_{i}: \mathcal{X}^{n+1} \times[0, \infty) \rightarrow[0,1]$ is a mapping such that

$$
\begin{aligned}
\lim _{j \rightarrow \infty} & \Psi_{i}\left(\lambda_{1}^{j} x_{1}, \ldots, x_{i}, x_{i}^{\prime}, x_{i+1}, \ldots, x_{n},\left|\lambda_{1}\right|^{2 j} t\right) \\
& =\cdots \\
& =\lim _{j \rightarrow \infty} \Psi_{i}\left(x_{1}, \ldots, x_{i-2}, \lambda_{1}^{j} x_{i-1}, x_{i}, x_{i}^{\prime}, x_{i+1}, \ldots, x_{n},\left|\lambda_{1}\right|^{2 j} t\right) \\
& =\lim _{j \rightarrow \infty} \Psi_{i}\left(x_{1}, \ldots, x_{i-1}, \lambda_{1}^{j} x_{i}, \lambda_{1}^{j} x_{i}^{\prime}, x_{i+1}, \ldots, x_{n},\left|\lambda_{1}\right|^{2 j} t\right) \\
& =\lim _{j \rightarrow \infty} \Psi_{i}\left(x_{1}, \ldots, x_{i}, x_{i}^{\prime}, \lambda_{1}^{j} x_{i+1}, x_{i+2}, \ldots, x_{n},\left|\lambda_{1}\right|^{2 j} t\right)=\ldots \\
& =\lim _{j \rightarrow \infty} \Psi_{i}\left(x_{1}, \ldots, x_{i}, x_{i}^{\prime}, x_{i+1}, \ldots, x_{n-1}, \lambda_{1}^{j} x_{n},\left|\lambda_{1}\right|^{2 j} t\right)=1
\end{aligned}
$$

and

$$
\begin{aligned}
\lim _{k \rightarrow \infty} T_{j=k}^{\infty} \Psi_{i}\left(x_{1}, \ldots, x_{i-1}, \lambda_{1}^{j} x_{i}, \lambda_{1}^{j} x_{i}, x_{i+1}, \ldots, x_{n},\left|\lambda_{1}\right|^{2 j+2} t\right) \\
\quad=\lim _{k \rightarrow \infty} T_{j=1}^{\infty} \Psi_{i}\left(x_{1}, \ldots, x_{i-1}, \lambda_{1}^{k+j-1} x_{i}, \lambda_{1}^{k+j-1} x_{i}, x_{i+1}, \ldots, x_{n},\left|\lambda_{1}\right|^{2 k+2 j} t\right)=1
\end{aligned}
$$

for all $x_{1}, \ldots, x_{i}, x_{i}^{\prime}, x_{i+1}, \ldots, x_{n} \in \mathcal{X}$ and $t>0$. Iff $: \mathcal{X}^{n} \rightarrow \mathcal{Y}$ is a mapping satisfying (3.3) and

$$
\begin{aligned}
& N\left(f\left(x_{1}, \ldots, x_{i-1}, a\left(x_{i}+x_{i}^{\prime}\right), x_{i+1}, \ldots, x_{n}\right)+f\left(x_{1}, \ldots, x_{i-1}, a\left(x_{i}-x_{i}^{\prime}\right), x_{i+1}, \ldots, x_{n}\right)\right. \\
& \left.\quad-2 a^{2}\left[f\left(x_{1}, \ldots, x_{n}\right)+f\left(x_{1}, \ldots, x_{i-1}, x_{i}^{\prime}, x_{i+1}, \ldots, x_{n}\right)\right], t\right) \\
& \geq \Psi_{i}\left(x_{1}, \ldots, x_{i}, x_{i}^{\prime}, x_{i+1}, \ldots, x_{n}, t\right)
\end{aligned}
$$

for all $x_{1}, \ldots, x_{i}, x_{i}^{\prime}, x_{i+1}, \ldots, x_{n} \in \mathcal{X}, i \in\{1,2, \ldots, n\}$ and $t \in[0, \infty)$, then for every $i \in$ $\{1,2, \ldots, n\}$ there exists a unique general multi-Euler-Lagrange quadratic mapping $Q_{i}$ : $\mathcal{X}^{n} \rightarrow \mathcal{Y}$ satisfying the functional equation (1.5) and such that

$$
\begin{aligned}
& N\left(f\left(x_{1}, \ldots, x_{n}\right)-Q_{i}\left(x_{1}, \ldots, x_{n}\right), t\right) \\
& \quad \geq T_{j=1}^{\infty} \Psi_{i}\left(x_{1}, \ldots, x_{i-1}, \lambda_{1}^{j-1} x_{i}, \lambda_{1}^{j-1} x_{i}, x_{i+1}, \ldots, x_{n},\left|\lambda_{1}\right|^{2 j} t\right)
\end{aligned}
$$

for all $x_{1}, \ldots, x_{n} \in \mathcal{X}$ and $t>0$.

Proof Fix $x_{1}, \ldots, x_{n} \in \mathcal{X}, j \in \mathbb{N} \cup\{0\}, i \in\{1,2, \ldots, n\}$ and $t>0$. Putting $x_{i}^{\prime}=x_{i}$ in (3.11), we get

$$
\begin{aligned}
& N\left(\frac{1}{\lambda_{1}^{2}} f\left(x_{1}, \ldots, x_{i-1}, \lambda_{1} x_{i}, x_{i+1}, \ldots, x_{n}\right)-f\left(x_{1}, \ldots, x_{n}\right), t\right) \\
& \quad \geq \Psi_{i}\left(x_{1}, \ldots, x_{i}, x_{i}, x_{i+1}, \ldots, x_{n},\left|\lambda_{1}\right|^{2} t\right) .
\end{aligned}
$$

Hence,

$$
\begin{aligned}
& N\left(\frac{1}{\lambda_{1}^{2(j+1)}} f\left(x_{1}, \ldots, x_{i-1}, \lambda_{1}^{j+1} x_{i}, x_{i+1}, \ldots, x_{n}\right)-\frac{1}{\lambda_{1}^{2 j}} f\left(x_{1}, \ldots, x_{i-1}, \lambda_{1}^{j} x_{i}, x_{i+1}, \ldots, x_{n}\right), t\right) \\
& \quad \geq \Psi_{i}\left(x_{1}, \ldots, x_{i-1}, \lambda_{1}^{j} x_{i}, \lambda_{1}^{j} x_{i}, x_{i+1}, \ldots, x_{n},\left|\lambda_{1}\right|^{2 j+2} t\right) .
\end{aligned}
$$


Therefore one can get

$$
\begin{aligned}
& N\left(\frac{1}{\lambda_{1}^{2(k+p)}} f\left(x_{1}, \ldots, x_{i-1}, \lambda_{1}^{k+p} x_{i}, x_{i+1}, \ldots, x_{n}\right)-\frac{1}{\lambda_{1}^{2 k}} f\left(x_{1}, \ldots, x_{i-1}, \lambda^{k} x_{i}, x_{i+1}, \ldots, x_{n}\right), t\right) \\
& \geq T_{j=k}^{k+p-1} N\left(\frac{1}{\lambda_{1}^{2(j+1)}} f\left(x_{1}, \ldots, x_{i-1}, \lambda_{1}^{j+1} x_{i}, x_{i+1}, \ldots, x_{n}\right)\right. \\
& \left.\quad-\frac{1}{\lambda_{1}^{2 j}} f\left(x_{1}, \ldots, x_{i-1}, \lambda_{1}^{j} x_{i}, x_{i+1}, \ldots, x_{n}\right), t\right) \\
& \geq T_{j=k}^{k+p-1} \Psi_{i}\left(x_{1}, \ldots, x_{i-1}, \lambda_{1}^{j} x_{i}, \lambda_{1}^{j} x_{i}, x_{i+1}, \ldots, x_{n},\left|\lambda_{1}\right|^{2 j+2} t\right),
\end{aligned}
$$

and thus by (3.10) it follows that $\left\{\frac{1}{\lambda_{1}^{2 j}} f\left(x_{1}, \ldots, x_{i-1}, \lambda_{1}^{j} x_{i}, x_{i+1}, \ldots, x_{n}\right)\right\}_{j \in \mathbb{N}}$ is a Cauchy sequence in a complete non-Archimedean fuzzy normed space. Hence, we can define a mapping $Q_{i}: \mathcal{X}^{n} \rightarrow \mathcal{Y}$ such that

$$
\lim _{j \rightarrow \infty} N\left(\frac{1}{\lambda_{1}^{2 j}} f\left(x_{1}, \ldots, x_{i-1}, \lambda_{1}^{j} x_{i}, x_{i+1}, \ldots, x_{n}\right)-Q_{i}\left(x_{1}, \ldots, x_{n}\right), t\right)=1 .
$$

Using (3.13) and induction, one can show that for any $k \in \mathbb{N}$ we have

$$
\begin{array}{r}
N\left(f\left(x_{1}, \ldots, x_{n}\right)-\frac{1}{\lambda_{1}^{2 k}} f\left(x_{1}, \ldots, x_{i-1}, \lambda_{1}^{k} x_{i}, x_{i+1}, \ldots, x_{n}\right), t\right) \\
\quad \geq T_{j=1}^{k} \Psi_{i}\left(x_{1}, \ldots, x_{i-1}, \lambda_{1}^{j-1} x_{i}, \lambda_{1}^{j-1} x_{i}, x_{i+1}, \ldots, x_{n},\left|\lambda_{1}\right|^{2 j} t\right) .
\end{array}
$$

Therefore,

$$
\begin{aligned}
N( & \left.f\left(x_{1}, \ldots, x_{n}\right)-Q_{i}\left(x_{1}, \ldots, x_{n}\right), t\right) \\
\geq & T\left(T_{j=1}^{k} \Psi_{i}\left(x_{1}, \ldots, x_{i-1}, \lambda_{1}^{j-1} x_{i}, \lambda_{1}^{j-1} x_{i}, x_{i+1}, \ldots, x_{n},\left|\lambda_{1}\right|^{2 j} t\right),\right. \\
& \left.N\left(\lambda_{1}^{-2 k} f\left(x_{1}, \ldots, x_{i-1}, \lambda_{1}^{k} x_{i}, x_{i+1}, \ldots, x_{n}\right)-Q_{i}\left(x_{1}, \ldots, x_{n}\right), t\right)\right) .
\end{aligned}
$$

Letting $k \rightarrow \infty$ in this inequality, we obtain (3.12). The rest of the proof of this theorem is omitted as being similar to the corresponding that of Theorem 3.1.

Let $(\mathcal{Y}, N, T)$ be a complete non-Archimedean fuzzy normed space over a non-Archimedean field $\mathbb{K}$. In any such space, a sequence $\left\{x_{k}\right\}_{k \in \mathbb{N}}$ is Cauchy if and only if $\left\{x_{k+1}-x_{k}\right\}_{k \in \mathbb{N}}$ converges to zero. Analysis similar to that in the proof of Theorem 3.2 gives the following.

Theorem 3.3 Let $\mathbb{K}$ be a non-Archimedean field, $\mathcal{X}$ be a vector space over $\mathbb{K}$ and $(\mathcal{Y}, N, T)$ be a complete non-Archimedean fuzzy normed space over $\mathbb{K}$. Assume also that, for every $i \in\{1,2, \ldots, n\}, \Psi_{i}: \mathcal{X}^{n+1} \times[0, \infty) \rightarrow[0,1]$ is a mapping such that (3.9) holds and

$$
\lim _{k \rightarrow \infty} T_{j=1}^{\infty} \Psi_{i}\left(x_{1}, \ldots, x_{i-1}, \lambda_{1}^{k+j-1} x_{i}, \lambda_{1}^{k+j-1} x_{i}, x_{i+1}, \ldots, x_{n},\left|\lambda_{1}\right|^{2 k+2 j} t\right)=1
$$

for all $x_{1}, \ldots, x_{i}, x_{i}^{\prime}, x_{i+1}, \ldots, x_{n} \in \mathcal{X}$ and $t>0$. If $: \mathcal{X}^{n} \rightarrow \mathcal{Y}$ is a mapping satisfying (3.3) and (3.11), then for every $i \in\{1,2, \ldots, n\}$ there exists a unique general multi-Euler-Lagrange quadratic mapping $Q_{i}: \mathcal{X}^{n} \rightarrow \mathcal{Y}$ satisfying (1.5) and (3.12). 
Remark 3.4 Let $a, b \in \mathbb{N}$ and $\mathcal{X}$ be a commutative group, Theorems 3.1-3.3 also hold. For $a=1$, consider the non-Archimedean fuzzy normed space $\left(\mathcal{Y}, N_{1}, T_{M}\right)$ defined as in Example 2.6, Theorem 3.3 yields Theorem 2 in [7]. If $a=b= \pm \frac{1}{\sqrt{2}} \in \mathbb{K}$, then $\lambda_{1}=2 a=$ $\pm \sqrt{2} \neq 1$ in Theorems 3.2-3.3 and $\lambda=a^{2}+b^{2}=1$ which is a singular case $\lambda=1$ of Theorem 3.1.

Analysis similar to that in the proof of Theorem 3.1 gives the following.

Theorem 3.5 Let $\mathbb{K}$ be a valued field, $\mathcal{X}$ be a vector space over $\mathbb{K}$ and $(\mathcal{Y}, N, T)$ be a complete non-Archimedean fuzzy normed space over $\mathbb{K}$. Assume also that, for every $i \in\{1,2, \ldots, n\}, \Psi_{i}: \mathcal{X}^{n+1} \times[0, \infty) \rightarrow[0,1]$ is a mapping such that

$$
\begin{aligned}
& \lim _{j \rightarrow \infty} \Psi_{i}\left(\lambda^{-j} x_{1}, \ldots, x_{i}, x_{i}^{\prime}, x_{i+1}, \ldots, x_{n},|\lambda|^{-2 j} t\right) \\
& \quad=\cdots \\
& \quad=\lim _{j \rightarrow \infty} \Psi_{i}\left(x_{1}, \ldots, x_{i-2}, \lambda^{-j} x_{i-1}, x_{i}, x_{i}^{\prime}, x_{i+1}, \ldots, x_{n},|\lambda|^{-2 j} t\right) \\
& \quad=\lim _{j \rightarrow \infty} \Psi_{i}\left(x_{1}, \ldots, x_{i-1}, \lambda^{-j} x_{i}, \lambda^{-j} x_{i}^{\prime}, x_{i+1}, \ldots, x_{n},|\lambda|^{-2 j} t\right) \\
& \quad=\lim _{j \rightarrow \infty} \Psi_{i}\left(x_{1}, \ldots, x_{i}, x_{i}^{\prime}, \lambda^{-j} x_{i+1}, x_{i+2}, \ldots, x_{n},|\lambda|^{-2 j} t\right)=\cdots \\
& \quad=\lim _{j \rightarrow \infty} \Psi_{i}\left(x_{1}, \ldots, x_{i}, x_{i}^{\prime}, x_{i+1}, \ldots, x_{n-1}, \lambda^{-j} x_{n},|\lambda|^{-2 j} t\right)=1
\end{aligned}
$$

and

$$
\begin{aligned}
\lim _{k \rightarrow \infty} & T_{j=k}^{\infty} T\left(\Psi_{i}\left(x_{1}, \ldots, x_{i-1}, \lambda^{-j-1} x_{i}, 0, x_{i+1}, \ldots, x_{n},|\lambda|^{-2 j-1} t\right),\right. \\
& \left.\Psi_{i}\left(x_{1}, \ldots, x_{i-1}, a \lambda^{-j-1} x_{i}, b \lambda^{-j-1} x_{i}, x_{i+1}, \ldots, x_{n},|\lambda|^{-2 j} t\right)\right) \\
= & \lim _{k \rightarrow \infty} T_{j=1}^{\infty} T\left(\Psi_{i}\left(x_{1}, \ldots, x_{i-1}, \lambda^{-k-j-1} x_{i}, 0, x_{i+1}, \ldots, x_{n},|\lambda|^{-2 k-2 j-1} t\right),\right. \\
& \left.\Psi_{i}\left(x_{1}, \ldots, x_{i-1}, a \lambda^{-k-j-1} x_{i}, b \lambda^{-k-j-1} x_{i}, x_{i+1}, \ldots, x_{n},|\lambda|^{-2 k-2 j} t\right)\right)=1
\end{aligned}
$$

for all $x_{1}, \ldots, x_{i}, x_{i}^{\prime}, x_{i+1}, \ldots, x_{n} \in \mathcal{X}$ and $t>0$. If $: \mathcal{X}^{n} \rightarrow \mathcal{Y}$ is a mapping satisfying (3.3) and (3.4), then for every $i \in\{1,2, \ldots, n\}$ there exists a unique general multi-Euler-Lagrange quadratic mapping $Q_{i}: \mathcal{X}^{n} \rightarrow \mathcal{Y}$ such that

$$
\begin{aligned}
N\left(f\left(x_{1}, \ldots, x_{n}\right)-Q_{i}\left(x_{1}, \ldots, x_{n}\right), t\right) \\
\geq T_{j=1}^{\infty} T\left(\Psi_{i}\left(x_{1}, \ldots, x_{i-1}, \lambda^{-j-1} x_{i}, 0, x_{i+1}, \ldots, x_{n},|\lambda|^{-2 j-1} t\right),\right. \\
\left.\quad \Psi_{i}\left(x_{1}, \ldots, x_{i-1}, a \lambda^{-j-1} x_{i}, b \lambda^{-j-1} x_{i}, x_{i+1}, \ldots, x_{n},|\lambda|^{-2 j} t\right)\right)
\end{aligned}
$$

for all $x_{1}, \ldots, x_{n} \in \mathcal{X}$ and $t>0$.

Corollary 3.6 Let $\mathbb{K}$ be a non-Archimedean field with $0<|\lambda|<1, \mathcal{X}$ be a normed space over $\mathbb{K}$ and let $(\mathcal{Y}, N, T)$ be a complete non-Archimedean fuzzy normed space over $\mathbb{K}$ under a $t$-norm $T \in \mathcal{H}$. Assume also that $\delta>0$ and $\alpha:[0, \infty) \rightarrow[0, \infty)$ is a function such that $\alpha\left(|\lambda|^{-1}\right)<|\lambda|^{-1}$ and $\alpha\left(|\lambda|^{-1} t\right) \leq \alpha\left(|\lambda|^{-1}\right) \alpha(t)$ for all $t \in[0, \infty)$. 
Iff $: \mathcal{X}^{n} \rightarrow \mathcal{Y}$ is a mapping satisfying (3.3) and

$$
\begin{aligned}
& N\left(f\left(x_{1}, \ldots, x_{i-1}, a x_{i}+b x_{i}^{\prime}, x_{i+1}, \ldots, x_{n}\right)+f\left(x_{1}, \ldots, x_{i-1}, b x_{i}-a x_{i}^{\prime}, x_{i+1}, \ldots, x_{n}\right)\right. \\
& \left.\quad-\left(a^{2}+b^{2}\right)\left[f\left(x_{1}, \ldots, x_{n}\right)+f\left(x_{1}, \ldots, x_{i-1}, x_{i}^{\prime}, x_{i+1}, \ldots, x_{n}\right)\right], t\right) \\
& \geq \frac{t}{t+\delta \alpha\left(\left\|x_{i}\right\|\right) \alpha\left(\left\|x_{i}^{\prime}\right\|\right)}
\end{aligned}
$$

for all $x_{1}, \ldots, x_{i}, x_{i}^{\prime}, x_{i+1}, \ldots, x_{n} \in \mathcal{X}, i \in\{1,2, \ldots, n\}$ and $t \in[0, \infty)$, then for every $i \in$ $\{1,2, \ldots, n\}$ there exists a unique general multi-Euler-Lagrange quadratic mapping $Q_{i}$ : $\mathcal{X}^{n} \rightarrow \mathcal{Y}$ such that

$$
\begin{aligned}
& N\left(f\left(x_{1}, \ldots, x_{n}\right)-Q_{i}\left(x_{1}, \ldots, x_{n}\right), t\right) \\
& \quad \geq T_{j=1}^{\infty} T\left(\frac{t}{t+\delta|\lambda|^{2 j+1} \alpha\left(|\lambda|^{-1}\right)^{j+1} \alpha\left(\left\|x_{i}\right\|\right) \alpha(0)}, \frac{t}{t+\delta|\lambda|^{2 j} \alpha\left(|\lambda|^{-1}\right)^{2 j+2} \alpha\left(\left\|a x_{i}\right\|\right) \alpha\left(\left\|b x_{i}\right\|\right)}\right)
\end{aligned}
$$

for all $x_{1}, \ldots, x_{n} \in \mathcal{X}$ and $t>0$.

Proof Fix $i \in\{1,2, \ldots, n\}, x_{1}, \ldots, x_{i}, x_{i}^{\prime}, x_{i+1}, \ldots, x_{n} \in \mathcal{X}$ and $t \in[0, \infty)$. Let $\Psi_{i}: \mathcal{X}^{n+1} \times$ $[0, \infty) \rightarrow[0,1]$ be defined by $\Psi_{i}\left(x_{1}, \ldots, x_{i}, x_{i}^{\prime}, x_{i+1}, \ldots, x_{n}, t\right):=\frac{t}{t+\delta \alpha\left(\left\|x_{i}\right\|\right) \alpha\left(\left\|x_{i}^{\prime}\right\|\right)}$. Then we can apply Theorem 3.5 to obtain the result.

Remark 3.7 Let $0<|\lambda|<1$ and $p \in(0,1)$. Then the mapping $\alpha:[0, \infty) \rightarrow[0, \infty)$ given by $\alpha(t):=t^{p}, t \in[0, \infty)$ satisfies $\alpha\left(|\lambda|^{-1}\right)<|\lambda|^{-1}$ and $\alpha\left(|\lambda|^{-1} t\right) \leq \alpha\left(|\lambda|^{-1}\right) \alpha(t)$ for all $t \in[0, \infty)$.

\section{Stability of the functional equation (1.1): a fixed point method}

Throughout this section, we prove the stability of Eq. (1.1) in complete non-Archimedean fuzzy normed spaces using the fixed point method.

Theorem 4.1 Let $\mathbb{K}$ be a valued field, $\mathcal{X}$ be a vector space over $\mathbb{K}$ and $(\mathcal{Y}, N, T)$ be a complete non-Archimedean fuzzy normed space over $\mathbb{K}$. Assume also that, for every $i \in\{1,2, \ldots, n\}, \Psi_{i}: \mathcal{X}^{n+1} \times[0, \infty) \rightarrow[0,1]$ is a mapping such that (3.1) holds and

$$
\begin{aligned}
& \Psi_{i}\left(x_{1}, \ldots, x_{i-1}, \lambda x_{i}, \lambda x_{i}, x_{i+1}, \ldots, x_{n},|\lambda|^{2} L_{i} t\right) \\
& \quad \geq \Psi_{i}\left(x_{1}, \ldots, x_{i}, x_{i}, x_{i+1}, \ldots, x_{n}, t\right), \quad x_{1}, \ldots, x_{n} \in \mathcal{X}
\end{aligned}
$$

for an $L_{i} \in(0,1)$. If $f: \mathcal{X}^{n} \rightarrow \mathcal{Y}$ is a mapping satisfying (3.3) and (3.4), then for every $i \in\{1,2, \ldots, n\}$ there exists a unique general multi-Euler-Lagrange quadratic mapping $Q_{i}: \mathcal{X}^{n} \rightarrow \mathcal{Y}$ such that

$$
\begin{aligned}
N( & \left.f\left(x_{1}, \ldots, x_{n}\right)-Q_{i}\left(x_{1}, \ldots, x_{n}\right), t\right) \\
\geq & T\left(\Psi_{i}\left(x_{1}, \ldots, x_{i}, 0, x_{i+1}, \ldots, x_{n},|\lambda|\left(1-L_{i}\right) t\right),\right. \\
& \left.\Psi_{i}\left(x_{1}, \ldots, x_{i-1}, a x_{i}, b x_{i}, x_{i+1}, \ldots, x_{n},|\lambda|^{2}\left(1-L_{i}\right) t\right)\right)
\end{aligned}
$$

for all $x_{1}, \ldots, x_{n} \in \mathcal{X}$ and $t>0$. 
Proof Fix an $i \in\{1,2, \ldots, n\}$. Consider the set $\Omega:=\left\{g: \mathcal{X}^{n} \rightarrow \mathcal{Y}\right\}$ and introduce the generalized metric on $\Omega$ :

$$
\begin{aligned}
d_{i}(g, h)= & \inf \left\{C \in[0, \infty]: N\left(g\left(x_{1}, \ldots, x_{n}\right)-h\left(x_{1}, \ldots, x_{n}\right), C t\right)\right. \\
& \geq T\left(\Psi_{i}\left(x_{1}, \ldots, x_{i}, 0, x_{i+1}, \ldots, x_{n},|\lambda| t\right),\right. \\
& \left.\Psi_{i}\left(x_{1}, \ldots, x_{i-1}, a x_{i}, b x_{i}, x_{i+1}, \ldots, x_{n},|\lambda|^{2} t\right)\right), \\
& \left.x_{1}, \ldots, x_{n} \in \mathcal{X}, t>0\right\}, \quad g, h \in \Omega .
\end{aligned}
$$

A standard verification (see for instance [19]) shows that $\left(\Omega, d_{i}\right)$ is a complete generalized metric space. We now define an operator $J_{i}: \Omega \rightarrow \Omega$ by

$$
J_{i} g\left(x_{1}, \ldots, x_{n}\right)=\frac{1}{\lambda^{2}} g\left(x_{1}, \ldots, x_{i-1}, \lambda x_{i}, x_{i+1}, \ldots, x_{n}\right), \quad g \in \Omega, x_{1}, \ldots, x_{n} \in \mathcal{X}
$$

Let $g, h \in \Omega$ and $C_{g, h} \in[0, \infty]$ with $d_{i}(g, h) \leq C_{g, h}$. Then

$$
\begin{aligned}
N\left(g\left(x_{1}, \ldots, x_{n}\right)-h\left(x_{1}, \ldots, x_{n}\right), C_{g, h} t\right) \geq & T\left(\Psi_{i}\left(x_{1}, \ldots, x_{i}, 0, x_{i+1}, \ldots, x_{n},|\lambda| t\right),\right. \\
& \left.\Psi_{i}\left(x_{1}, \ldots, x_{i-1}, a x_{i}, b x_{i}, x_{i+1}, \ldots, x_{n},|\lambda|^{2} t\right)\right),
\end{aligned}
$$

which together with (4.1) gives

$$
\begin{aligned}
N\left(J_{i} g\left(x_{1}, \ldots, x_{n}\right)-J_{i} h\left(x_{1}, \ldots, x_{n}\right), t\right) \geq & T\left(\Psi_{i}\left(x_{1}, \ldots, x_{i}, 0, x_{i+1}, \ldots, x_{n}, \frac{|\lambda| t}{L_{i} C_{g, h}}\right),\right. \\
& \left.\Psi_{i}\left(x_{1}, \ldots, x_{i-1}, a x_{i}, b x_{i}, x_{i+1}, \ldots, x_{n}, \frac{|\lambda|^{2} t}{L_{i} C_{g, h}}\right)\right),
\end{aligned}
$$

and consequently, $d_{i}\left(J_{i} g, J_{i} h\right) \leq L_{i} C_{g, h}$, which means that the operator $J_{i}$ is strictly contractive. Moreover, from (3.8) it follows that

$$
\begin{aligned}
N\left(J_{i} f\left(x_{1}, \ldots, x_{n}\right)-f\left(x_{1}, \ldots, x_{n}\right), t\right) \geq & T\left(\Psi_{i}\left(x_{1}, \ldots, x_{i}, 0, x_{i+1}, \ldots, x_{n},|\lambda| t\right),\right. \\
& \left.\Psi_{i}\left(x_{1}, \ldots, x_{i-1}, a x_{i}, b x_{i}, x_{i+1}, \ldots, x_{n},|\lambda|^{2} t\right)\right)
\end{aligned}
$$

and thus $d_{i}\left(J_{i} f, f\right) \leq 1<\infty$. Therefore, by Theorem 2.8, $J_{i}$ has a unique fixed point $Q_{i}$ : $\mathcal{X}^{n} \rightarrow \mathcal{Y}$ in the set $\Omega^{\prime \prime}=\{g \in \Omega: d(f, g)<\infty\}$ such that

$$
\frac{1}{\lambda^{2}} Q_{i}\left(x_{1}, \ldots, x_{i-1}, \lambda x_{i}, x_{i+1}, \ldots, x_{n}\right)=Q_{i}\left(x_{1}, \ldots, x_{n}\right)
$$

and

$$
Q_{i}\left(x_{1}, \ldots, x_{n}\right)=\lim _{j \rightarrow \infty} \frac{1}{\lambda^{2 j}} f\left(x_{1}, \ldots, x_{i-1}, \lambda^{j} x_{i}, x_{i+1}, \ldots, x_{n}\right) .
$$

Furthermore, from the fact that $f \in \Omega^{*}$, Theorem 2.8 , and $d_{i}\left(J_{i} f, f\right) \leq 1$, we get

$$
d_{i}\left(f, Q_{i}\right) \leq \frac{1}{1-L_{i}} d_{i}\left(J_{i} f, f\right) \leq \frac{1}{1-L_{i}}
$$


and (4.2) follows. Similar to the proof of Theorem 3.1, one can prove that the mapping $Q_{i}$ is also general multi-Euler-Lagrange quadratic.

Let us finally assume that $Q_{i}^{\prime}: \mathcal{X}^{n} \rightarrow \mathcal{Y}$ is a general multi-Euler-Lagrange quadratic mapping satisfying condition (4.2). Then $Q_{i}^{\prime}$ fulfills (4.3), and therefore, it is a fixed point of the operator $J_{i}$. Moreover, by (4.2), we have $d_{i}\left(f, Q_{i}^{\prime}\right) \leq \frac{1}{1-L_{i}}<\infty$, and consequently $Q_{i}^{\prime} \in \Omega^{*}$. Theorem 2.8 shows that $Q_{i}^{\prime}=Q_{i}$.

Similar to Theorem 4.1, one can prove the following result.

Theorem 4.2 Let $\mathbb{K}$ be a valued field, $\mathcal{X}$ be a vector space over $\mathbb{K}$ and $(\mathcal{Y}, N, T)$ be a complete non-Archimedean fuzzy normed space over $\mathbb{K}$. Assume also that, for every $i \in\{1,2, \ldots, n\}, \Psi_{i}: \mathcal{X}^{n+1} \times[0, \infty) \rightarrow[0,1]$ is a mapping such that (3.14) holds and

$$
\begin{aligned}
& \Psi_{i}\left(x_{1}, \ldots, x_{i-1}, \lambda^{-1} x_{i}, \lambda^{-1} x_{i}, x_{i+1}, \ldots, x_{n},|\lambda|^{-2} L_{i} t\right) \\
& \quad \geq \Psi_{i}\left(x_{1}, \ldots, x_{i}, x_{i}, x_{i+1}, \ldots, x_{n}, t\right), \quad x_{1}, \ldots, x_{n} \in \mathcal{X}
\end{aligned}
$$

for an $L_{i} \in(0,1)$. If $f: \mathcal{X}^{n} \rightarrow \mathcal{Y}$ is a mapping satisfying (3.3) and (3.4), then for every $i \in\{1,2, \ldots, n\}$ there exists a unique general multi-Euler-Lagrange quadratic mapping $Q_{i}: \mathcal{X}^{n} \rightarrow \mathcal{Y}$ such that

$$
\begin{aligned}
N\left(f\left(x_{1}, \ldots, x_{n}\right)-Q_{i}\left(x_{1}, \ldots, x_{n}\right), t\right) \geq & T\left(\Psi_{i}\left(x_{1}, \ldots, x_{i}, 0, x_{i+1}, \ldots, x_{n},|\lambda|\left(L_{i}^{-1}-1\right) t\right)\right. \\
& \left.\Psi_{i}\left(x_{1}, \ldots, x_{i-1}, a x_{i}, b x_{i}, x_{i+1}, \ldots, x_{n},|\lambda|^{2}\left(L_{i}^{-1}-1\right) t\right)\right)
\end{aligned}
$$

for all $x_{1}, \ldots, x_{n} \in \mathcal{X}$ and $t>0$.

Remark 4.3 Similar to the proof of Corollary 3.6, one can deduce from Theorem 4.2 an analog of Corollary 3.6.

As applications of Theorems 4.1 and 4.2 , we get the following corollaries.

Corollary 4.4 Let $\mathcal{X}$ be a real normed space, $\mathcal{Y}$ be a real Banach space and $\left(\mathcal{Y}, N, T_{P}\right)$ be the complete non-Archimedean fuzzy normed space defined as in the second example in the preliminaries. Let $\delta, r, p, q \in(0, \infty)$ such that $r, s:=p+q \in(2, \infty)$, or $r, s \in(0,2)$. If $f: \mathcal{X}^{n} \rightarrow \mathcal{Y}$ is a mapping satisfying (3.3) and (3.4), where

$$
\Psi_{i}\left(x_{1}, \ldots, x_{i}, x_{i}^{\prime}, x_{i+1}, \ldots, x_{n}, t\right):=\frac{t}{t+\delta\left[\left\|x_{1}\right\|^{r} \cdots\left\|x_{i-1}\right\|^{r}\left(\left\|x_{i}\right\|^{p}\left\|x_{i}^{\prime}\right\|^{q}\right)\left\|x_{i+1}\right\|^{r} \cdots\left\|x_{n}\right\|^{r}\right]},
$$

then for every $i \in\{1,2, \ldots, n\}$ there exists a unique general multi-Euler-Lagrange quadratic mapping $Q_{i}: \mathcal{X}^{n} \rightarrow \mathcal{Y}$ such that

$$
\begin{aligned}
& e^{-\frac{\left\|f\left(x_{1}, \ldots, x_{n}\right)-Q_{i}\left(x_{1}, \ldots, x_{n}\right)\right\|}{t}} \\
& \quad \geq \frac{\left|\lambda^{s}-\lambda^{2}\right| t}{\left|\lambda^{s}-\lambda^{2}\right| t+\delta|a|^{p}|b|^{q}\left(\left\|x_{1}\right\|^{r} \cdots\left\|x_{i-1}\right\|^{r}\left\|x_{i}\right\|^{s}\left\|x_{i+1}\right\|^{r} \cdots\left\|x_{n}\right\|^{r}\right)}
\end{aligned}
$$

for all $x_{1}, \ldots, x_{n} \in \mathcal{X}$ and $t>0$. 
Proof Fix $i \in\{1,2, \ldots, n\}, x_{1}, \ldots, x_{i}, x_{i}^{\prime}, x_{i+1}, \ldots, x_{n} \in \mathcal{X}, t \in[0, \infty)$ and assume that $\lambda>1$, $r, s \in(2, \infty)$ (the same arguments apply to the case where $\lambda<1, r, s \in(0,2))$. Then we can choose $L_{i}=\lambda^{2-s}<1$ and apply Theorem 4.2 to obtain the result. For $\lambda>1, r, s \in(0,2)$, or $\lambda<1, r, s \in(2, \infty)$, the corollary follows from Theorem 4.1 .

Corollary 4.5 Let $\mathcal{X}$ be a real normed space and $\mathcal{Y}$ be a real Banach space (or $\mathcal{X}$ be a nonArchimedean normed space and $\mathcal{Y}$ be a complete non-Archimedean normed space over a non-Archimedean field $\mathbb{K}$, respectively). Let $\delta>0$ and $r \in(0,2) \cup(2, \infty)$. Iff: $\mathcal{X}^{n} \rightarrow \mathcal{Y}$ is a mapping satisfying (3.3) and

$$
\begin{aligned}
& \| f\left(x_{1}, \ldots, x_{i-1}, a x_{i}+b x_{i}^{\prime}, x_{i+1}, \ldots, x_{n}\right)+f\left(x_{1}, \ldots, x_{i-1}, b x_{i}-a x_{i}^{\prime}, x_{i+1}, \ldots, x_{n}\right), \\
& \quad-\left(a^{2}+b^{2}\right)\left[f\left(x_{1}, \ldots, x_{n}\right)+f\left(x_{1}, \ldots, x_{i-1}, x_{i}^{\prime}, x_{i+1}, \ldots, x_{n}\right)\right] \| \\
& \leq \delta\left[\left\|x_{1}\right\|^{r} \cdots\left\|x_{i-1}\right\|^{r}\left(\left\|x_{i}\right\|^{r}+\left\|x_{i}^{\prime}\right\|^{r}\right)\left\|x_{i+1}\right\|^{r} \cdots\left\|x_{n}\right\|^{r}\right]
\end{aligned}
$$

then for every $i \in\{1,2, \ldots, n\}$ there exists a unique general multi-Euler-Lagrange quadratic mapping $Q_{i}: \mathcal{X}^{n} \rightarrow \mathcal{Y}$ such that

$$
\left\|f\left(x_{1}, \ldots, x_{n}\right)-Q_{i}\left(x_{1}, \ldots, x_{n}\right)\right\| \leq \frac{\max \left\{|\lambda|,|a|^{r}+|b|^{r}\right\} \delta\left(\left\|x_{1}\right\|^{r} \cdots\left\|x_{n}\right\|^{r}\right)}{\left.|| \lambda\right|^{r}-|\lambda|^{2} \mid}
$$

for all $x_{1}, \ldots, x_{n} \in \mathcal{X}$

Proof Consider the non-Archimedean fuzzy normed space $\left(\mathcal{Y}, N_{1}, T_{M}\right)$ defined as in the first example in the preliminaries, $\Psi_{i}$ be defined by

$$
\Psi_{i}\left(x_{1}, \ldots, x_{i}, x_{i}^{\prime}, x_{i+1}, \ldots, x_{n}, t\right):=\frac{t}{t+\delta\left[\left\|x_{1}\right\|^{r} \cdots\left\|x_{i-1}\right\|^{r}\left(\left\|x_{i}\right\|^{r}+\left\|x_{i}^{\prime}\right\|^{r}\right)\left\|x_{i+1}\right\|^{r} \cdots\left\|x_{n}\right\|^{r}\right]},
$$

and apply Theorems 4.1 and 4.2.

The following example shows that the Hyers-Ulam stability problem for the case of $r=2$ was excluded in Corollary 4.5.

Example 4.6 Let $\phi: \mathbb{C} \rightarrow \mathbb{C}$ be defined by

$$
\phi(x)= \begin{cases}x^{2}, & \text { for }|x|<1 \\ 1, & \text { for }|x| \geq 1\end{cases}
$$

Consider the function $f: \mathbb{C} \rightarrow \mathbb{C}$ be defined by

$$
f(x)=\sum_{j=0}^{\infty} \frac{\phi\left(\alpha^{j} x\right)}{\alpha^{2 j}}
$$

for all $x \in \mathbb{C}$, where $\alpha>\max \{|a|,|b|, 1\}$. Then $f$ satisfies the functional inequality

$$
\begin{aligned}
& \left|f(a x+b y)+f(b x-a y)-\left(a^{2}+b^{2}\right)[f(x)+f(y)]\right| \\
& \quad \leq \frac{2 \alpha^{4}\left(|a|^{2}+|b|^{2}+1\right)}{\alpha^{2}-1}\left(|x|^{2}+|y|^{2}\right)
\end{aligned}
$$


for all $x, y \in \mathbb{C}$, but there do not exist a general multi-Euler-Lagrange quadratic function $Q: \mathbb{C} \rightarrow \mathbb{C}$ and a constant $d>0$ such that $|f(x)-Q(x)| \leq d|x|^{2}$ for all $x \in \mathbb{C}$.

It is clear that $f$ is bounded by $\frac{\alpha^{2}}{\alpha^{2}-1}$ on $\mathbb{C}$. If $|x|^{2}+|y|^{2}=0$ or $|x|^{2}+|y|^{2} \geq \frac{1}{\alpha^{2}}$, then

$$
\left|f(a x+b y)+f(b x-a y)-\left(a^{2}+b^{2}\right)[f(x)+f(y)]\right| \leq \frac{2 \alpha^{4}\left(|a|^{2}+|b|^{2}+1\right)}{\alpha^{2}-1}\left(|x|^{2}+|y|^{2}\right) .
$$

Now suppose that $0<|x|^{2}+|y|^{2}<\frac{1}{\alpha^{2}}$. Then there exists an integer $k \geq 1$ such that

$$
\frac{1}{\alpha^{2(k+2)}} \leq|x|^{2}+|y|^{2}<\frac{1}{\alpha^{2(k+1)}} .
$$

Hence

$$
\alpha^{l}|a x+b y|<1, \quad \alpha^{l}|b x-a y|<1, \quad \alpha^{l}|x|<1, \quad \alpha^{l}|y|<1
$$

for all $l=0,1, \ldots, k-1$. From the definition of $f$ and the inequality (4.6), we obtain that $f$ satisfies (4.5). Now, we claim that the functional equation (1.1) is not stable for $r=2$ in Corollary 4.5. Suppose, on the contrary, that there exist a general multi-Euler-Lagrange quadratic function $Q: \mathbb{C} \rightarrow \mathbb{C}$ and a constant $d>0$ such that $|f(x)-Q(x)| \leq d|x|^{2}$ for all $x \in \mathbb{C}$. Then there exists a constant $c \in \mathbb{C}$ such that $Q(x)=c x^{2}$ for all rational numbers $x$. So, we obtain that

$$
|f(x)| \leq(d+|c|)|x|^{2}
$$

for all rational numbers $x$. Let $s \in \mathbb{N}$ with $s+1>d+|c|$. If $x$ is a rational number in $\left(0, \alpha^{-s}\right)$, then $\alpha^{j} x \in(0,1)$ for all $j=0,1, \ldots, s$, and for this $x$ we get

$$
f(x)=\sum_{j=0}^{\infty} \frac{\phi\left(\alpha^{j} x\right)}{\alpha^{2 j}} \geq \sum_{j=0}^{s} \frac{\phi\left(\alpha^{j} x\right)}{\alpha^{2 j}}=(s+1) x^{2}>(d+|c|) x^{2},
$$

which contradicts (4.7).

Corollary 4.7 Let $\mathbb{K}$ be a non-Archimedean field with $0<|\lambda|<1, \mathcal{X}$ be a normed space over $\mathbb{K}$ and $\mathcal{Y}$ be a complete non-Archimedean normed space over $\mathbb{K}$. Let $\delta, p, q \in(0, \infty)$ such that $p+q \in(0,2)$. Iff $: \mathcal{X}^{n} \rightarrow \mathcal{Y}$ is a mapping satisfying (3.3) and

$$
\begin{aligned}
& \| f\left(x_{1}, \ldots, x_{i-1}, a x_{i}+b x_{i}^{\prime}, x_{i+1}, \ldots, x_{n}\right)+f\left(x_{1}, \ldots, x_{i-1}, b x_{i}-a x_{i}^{\prime}, x_{i+1}, \ldots, x_{n}\right), \\
& \quad-\left(a^{2}+b^{2}\right)\left[f\left(x_{1}, \ldots, x_{n}\right)+f\left(x_{1}, \ldots, x_{i-1}, x_{i}^{\prime}, x_{i+1}, \ldots, x_{n}\right)\right] \| \leq \delta\left(\left\|x_{i}\right\|^{p}\left\|x_{i}^{\prime}\right\|^{q}\right),
\end{aligned}
$$

then for every $i \in\{1,2, \ldots, n\}$ there exists a unique general multi-Euler-Lagrange quadratic mapping $Q_{i}: \mathcal{X}^{n} \rightarrow \mathcal{Y}$ such that

$$
\left\|f\left(x_{1}, \ldots, x_{n}\right)-Q_{i}\left(x_{1}, \ldots, x_{n}\right)\right\| \leq \frac{\delta|a|^{p}|b|^{q}\left\|x_{i}\right\|^{p+q}}{|\lambda|^{p+q}-|\lambda|^{2}}
$$

for all $x_{1}, \ldots, x_{n} \in \mathcal{X}$. 
Proof Fix $i \in\{1,2, \ldots, n\}, x_{1}, \ldots, x_{i}, x_{i}^{\prime}, x_{i+1}, \ldots, x_{n} \in \mathcal{X}$ and $t \in[0, \infty)$. Let $\Psi_{i}: \mathcal{X}^{n+1} \times$ $[0, \infty) \rightarrow[0,1]$ be defined by $\Psi_{i}\left(x_{1}, \ldots, x_{i}, x_{i}^{\prime}, x_{i+1}, \ldots, x_{n}, t\right):=\frac{t}{t+\delta\left(\left\|x_{i}\right\|^{\|}\left\|x_{i}^{\prime}\right\|^{q}\right)}$. Consider the non-Archimedean fuzzy normed space $\left(\mathcal{Y}, N_{1}, T_{M}\right)$ defined as in Example 2.6, and apply Theorem 4.2.

Corollary 4.8 Let $\mathcal{X}$ be a real normed space and $\mathcal{Y}$ be a real Banach space. Let $\delta, r, p, q \in$ $(0, \infty)$ such that $r, p+q \in(0,2)$, or $r, p+q \in(2, \infty)$. If $: \mathcal{X}^{n} \rightarrow \mathcal{Y}$ is a mapping satisfying (3.3) and

$$
\begin{aligned}
& \| f\left(x_{1}, \ldots, x_{i-1}, a x_{i}+b x_{i}^{\prime}, x_{i+1}, \ldots, x_{n}\right)+f\left(x_{1}, \ldots, x_{i-1}, b x_{i}-a x_{i}^{\prime}, x_{i+1}, \ldots, x_{n}\right), \\
& \quad-\left(a^{2}+b^{2}\right)\left[f\left(x_{1}, \ldots, x_{n}\right)+f\left(x_{1}, \ldots, x_{i-1}, x_{i}^{\prime}, x_{i+1}, \ldots, x_{n}\right)\right] \| \\
& \leq \delta\left[\left\|x_{1}\right\|^{r} \cdots\left\|x_{i-1}\right\|^{r}\left(\left\|x_{i}\right\|^{p}\left\|x_{i}^{\prime}\right\|^{q}\right)\left\|x_{i+1}\right\|^{r} \cdots\left\|x_{n}\right\|^{r}\right],
\end{aligned}
$$

then, for every $i \in\{1,2, \ldots, n\}$, there exists a unique general multi-Euler-Lagrange quadratic mapping $Q_{i}: \mathcal{X}^{n} \rightarrow \mathcal{Y}$ such that

$$
\begin{aligned}
& \left\|f\left(x_{1}, \ldots, x_{n}\right)-Q_{i}\left(x_{1}, \ldots, x_{n}\right)\right\| \\
& \leq \frac{\delta|a|^{p}|b|^{q}\left(\left\|x_{1}\right\|^{r} \cdots\left\|x_{i-1}\right\|^{r}\left\|x_{i}\right\|^{p+q}\left\|x_{i+1}\right\|^{r} \cdots\left\|x_{n}\right\|^{r}\right)}{\left|\lambda^{p+q}-\lambda^{2}\right|}
\end{aligned}
$$

for all $x_{1}, \ldots, x_{n} \in \mathcal{X}$

Proof Fix $i \in\{1,2, \ldots, n\}, x_{1}, \ldots, x_{i}, x_{i}^{\prime}, x_{i+1}, \ldots, x_{n} \in \mathcal{X}$ and $t \in[0, \infty)$. Let $\Psi_{i}: \mathcal{X}^{n+1} \times$ $[0, \infty) \rightarrow[0,1]$ be defined by

$$
\Psi_{i}\left(x_{1}, \ldots, x_{i}, x_{i}^{\prime}, x_{i+1}, \ldots, x_{n}, t\right):=\frac{t}{t+\delta\left[\left\|x_{1}\right\|^{r} \cdots\left\|x_{i-1}\right\|^{r}\left(\left\|x_{i}\right\|^{p}\left\|x_{i}^{\prime}\right\|^{q}\right)\left\|x_{i+1}\right\|^{r} \cdots\left\|x_{n}\right\|^{r}\right]}
$$

Consider the non-Archimedean fuzzy normed space $\left(\mathcal{Y}, N_{1}, T_{M}\right)$ defined as in Example 2.6, and apply Theorems 4.1 and 4.2 .

Remark 4.9 Theorems 4.1 and 4.2 can be regarded as a generalization of the classical stability result in the framework of normed spaces (see [14]). For $a=b=1$ and $n=1$, Corollary 4.8 yields the main theorem in [17]. The generalized Hyers-Ulam stability problem for the case of $r=p+q=2$ was excluded in Corollary 4.8 (see[10]).

Note that by (4.4) one can get

$$
\begin{aligned}
& \left\|f\left(x_{1}, \ldots, x_{n}\right)-Q_{i}\left(x_{1}, \ldots, x_{n}\right)\right\| \\
& \quad \leq \ln \left(1+\frac{\delta|a|^{p}|b|^{q}\left(\left\|x_{1}\right\|^{r} \cdots\left\|x_{i-1}\right\|^{r}\left\|x_{i}\right\|^{p+q}\left\|x_{i+1}\right\|^{r} \cdots\left\|x_{n}\right\|^{r}\right)}{\left|\lambda^{p+q}-\lambda^{2}\right| \cdot t}\right)^{t} .
\end{aligned}
$$

Letting $t \rightarrow \infty$ in this inequality, we obtain (4.9). Thus Corollary 4.8 is a singular case of Corollary 4.4. This study indeed presents a relationship between three various disciplines: the theory of non-Archimedean fuzzy normed spaces, the theory of stability of functional equations and the fixed point theory. 


\section{Competing interests}

The authors declare that they have no competing interests.

\section{Authors' contributions}

All authors carried out the proof. All authors conceived of the study and participated in its design and coordination. All authors read and approved the final manuscript.

\section{Author details}

${ }^{1}$ School of Mathematics, Beijing Institute of Technology, Beijing, 100081, P.R. China. ${ }^{2}$ Pedagogical Department E.E., Section of Mathematics and Informatics, National and Capodistrian University of Athens, 4, Agamemnonos Str., Aghia Paraskevi, Athens, 15342, Greece.

\section{Acknowledgements}

The first author was supported by the National Natural Science Foundation of China (NNSFC) (Grant No. 11171022).

Received: 24 May 2012 Accepted: 5 July 2012 Published: 23 July 2012

\section{References}

1. Agarwal, RP, Xu, B, Zhang, W: Stability of functional equations in single variable. J. Math. Anal. Appl. 288, $852-869$ (2003)

2. Aoki, T: On the stability of the linear transformation in Banach spaces. J. Math. Soc. Jpn. 2, 64-66 (1950)

3. Cho, YJ, Grabiec, M, Radu, V: On Nonsymmetric Topological and Probabilistic Structures. Nova Science Publishers, New York (2006)

4. Cho, YJ, Park, C, Saadati, R: Functional inequalities in non-Archimedean Banach spaces. Appl. Math. Lett. 23, 1238-1242 (2010)

5. Cho, YJ, Saadati, R: Lattictic non-Archimedean random stability of ACQ functional equation. Adv. Differ. Equ. 2011,31 (2011)

6. Ebadian, A, Ghobadipour, N, Gordji, ME: On the stability of a parametric additive functional equation in quasi-Banach spaces. Abstr. Appl. Anal. 2012, 235359 (2012)

7. Ciepliński, K: On the generalized Hyers-Ulam stability of multi-quadratic mappings. Comput. Math. Appl. 62, 3418-3426 (2011)

8. Diaz, JB, Margolis, B: A fixed point theorem of the alternative for the contractions on generalized complete metric space. Bull. Am. Math. Soc. 74, 305-309 (1968)

9. Găvruţa, P: A generalization of the Hyers-Ulam-Rassias stability of approximately additive mappings. J. Math. Anal. Appl. 184, 431-436 (1994)

10. Găvruţa, L, Găvruţa, P: On a problem of John M. Rassias concerning the stability in Ulam sense of Euler-Lagrange equation. In: Rassias, JM (ed.) Functional Equations, Difference Inequalities and Ulam Stability Notions (F.U.N.) Chapter 4, pp. 47-53. Nova Science Publishers, New York (2010)

11. Gordji, ME, Ghaemi, MB, Cho, YJ, Majani, H: A general system of Euler-Lagrange-type quadratic functional equations in Menger probabilistic non-Archimedean 2-normed spaces. Abstr. Appl. Anal. 2011, 208163 (2011)

12. Hadžić, O, Pap, E, Budincević, M: Countable extension of triangular norms and their applications to the fixed point theory in probabilistic metric spaces. Kybernetika 38, 363-381 (2002)

13. Hyers, DH: On the stability of the linear functional equation. Proc. Natl. Acad. Sci. USA 27, $222-224$ (1941)

14. Hyers, DH, Isac, G, Rassias, TM: Stability of Functional Equations in Several Variables. Birkhäuser, Basel (1998)

15. Kenary, HA, Rezaei, H, Talebzadeh, S, Park, C: Stability of the Jensen equation in C*-algebras: a fixed point approach. Adv. Differ. Equ. 2012, 17 (2012)

16. Kenary, HA, Lee, J, Park, C: Nonlinear approximation of an ACQ-functional equation in nan-spaces. Fixed Point Theory Appl. 2011,60 (2011)

17. Rassias, JM: On the stability of the Euler-Lagrange functional equation. Chin. J. Math. 20, 185-190 (1992)

18. Jung, SM: Hyers-Ulam-Rassias Stability of Functional Equations in Nonlinear Analysis. Springer, New York (2011)

19. Jung, SM: A fixed point approach to the stability of the equation $f(x+y)=\frac{f(x) f(y)}{f(x)+f(y)}$. Aust. J. Math. Anal. Appl. 6, $8(2009)$

20. Kannappan, Pl: Functional Equations and Inequalities with Applications. Springer, New York (2009)

21. Khrennikov, A: Non-Archimedean Analysis: Quantum Paradoxes, Dynamical Systems and Biological Models. Kluwer Academic, Dordrecht (1997)

22. Miheț, D: The stability of the additive Cauchy functional equation in non-Archimedean fuzzy normed spaces. Fuzzy Sets Syst. 161, 2206-2212 (2010)

23. Mirmostafaee, AK, Moslehian, MS: Stability of additive mappings in non-Archimedean fuzzy normed spaces. Fuzzy Sets Syst. 160, 1643-1652 (2009)

24. Park, C: Multi-quadratic mappings in Banach spaces. Proc. Am. Math. Soc. 131, 2501-2504 (2003)

25. Park, C: Fixed points and the stability of an AQCQ-functional equation in non-Archimedean normed spaces. Abstr. Appl. Anal. 2010, 849543 (2010)

26. Radu, V: The fixed point alternative and the stability of functional equations. Fixed Point Theory 4, 91-96 (2003)

27. Rassias, TM: On the stability of the linear mapping in Banach spaces. Proc. Am. Math. Soc. 72, 297-300 (1978)

28. Saadati, R, Cho, YJ, Vahidi, J: The stability of the quartic functional equation in various spaces. Comput. Math. Appl. 60 1994-2002 (2010)

29. Schweizer, B, Sklar, A: Probabilistic Metric Spaces. North-Holland, New York (1983)

30. Ulam, SM: A Collection of the Mathematical Problems. Interscience, New York (1960)

31. Xu, TZ, Rassias, JM, Xu, WX: Stability of a general mixed additive-cubic functional equation in non-Archimedean fuzzy normed spaces. J. Math. Phys. 51, 093508 (2010)

32. $\mathrm{Xu}, \mathrm{TZ}$ : Stability of multi-Jensen mappings in non-Archimedean normed spaces. J. Math. Phys. 53, 023507 (2012)

33. Xu, TZ: On the stability of multi-Jensen mappings in $\beta$-normed spaces. Appl. Math. Lett. 25, 1866-1870 (2012). doi:10.1016/j.aml.2012.02.049 
34. $\mathrm{Xu}, \mathrm{TZ}$, Rassias, JM: On the Hyers-Ulam stability of a general mixed additive and cubic functional equation in n-Banach spaces. Abstr. Appl. Anal. 2012, 926390 (2012)

35. Xu, TZ, Rassias, JM: A fixed point approach to the stability of an AQ-functional equation on $\beta$-Banach modules. Fixed Point Theory Appl. 2012, 32 (2012)

36. Alotaibi, A, Mohiuddine, SA: On the stability of a cubic functional equation in random 2-normed spaces. Adv. Differ. Equ. 2012, 39 (2012)

37. Mohiuddine, SA, Alotaibi, A: Fuzzy stability of a cubic functional equation via fixed point technique. Adv. Differ. Equ. 2012, 48 (2012)

doi:10.1186/1687-1847-2012-119

Cite this article as: Xu and Rassias: Stability of general multi-Euler-Lagrange quadratic functional equations in non-Archimedean fuzzy normed spaces. Advances in Difference Equations 2012 2012:119.

Submit your manuscript to a SpringerOpen ${ }^{\circ}$ journal and benefit from:

- Convenient online submission

- Rigorous peer review

- Immediate publication on acceptance

- Open access: articles freely available online

- High visibility within the field

- Retaining the copyright to your article

Submit your next manuscript at $>$ springeropen.com 